\title{
LA INFINITA APERTURA DE LA RAZÓN \\ CiENCIA MODERNA Y SENTIDO DEL MISTERIO
}

\begin{tabular}{c} 
Paolo Musso* \\
Universidad de la Insubria de Varese \\
\hline
\end{tabular}

Resumen: Según una opinión muy común, la ciencia moderna habría sido inventada por Galileo Galilei y René Descartes. De esta deriva que el racionalismo y el mecanicismo cartesianos sean partes esenciales de la ciencia como tal, mientras que en realidad su origen solo es filosófica, pues Descartes nunca fue un científico. Por tanto, en el Renacimiento no nació una nueva cultura (la modernidad) y una nueva idea de razón (el racionalismo), sino dos: una basada en el método experimental de Galileo y en una idea abierta de razón, y otra, opuesta, basada en la filosofía de Descartes y en una idea de razón cerrada que no cree posible encontrar la verdad en la experiencia. Palabras clave: Galileo, Descartes, ciencia, método, razón, modernidad, mecanicismo

* Paolo Musso es docente de Filosofía de la Ciencia en la Università dell'Insubria de Varese. Ha estudiado los problemas del realismo científico, de la interpretación filosófica del caos determinista, del problema de la complejidad y de la mecánica cuántica; de cuestiones metodológicas y éticas en medicina y, especialmente en los últimos años, de bioastronomía. Es miembro del SETI Permanent Committee de la International Academy of Astronautics (IAA), el máximo organismo científico a nivel mundial en el campo de la exploración del espacio. Ha publicado Rom Harré e il problema del realismo scientífico (Angeli, Milán 1993), Filosofia del caos (Angeli, Milán 1997), Convivere con la bomba. Dalla bioetica alla biopolitica (ESI, Nápoles 2001) y (como editor) Il dolore e la medicina. Alla ricerca di senso e di cure (SEF, Firenze 2005), además de numerosos ensayos y artículos. 
PAOLO MUSSO

Abstract: According to a very common opinion, modern science was created by Galileo Galilei and René Descartes. This implies Cartesian rationalism and mechanicalism to be essential elements of science as such, while in reality their origin is only philosophical, since Descartes never was a scientist. So, in the Renaissence they were not born one new culture (the modernism) and one new idea of reason (the rationalism), but two: the first based on Galileo's experimental method and an open idea of reason, and the second, completely opposite, baesd on Descartes's philosophy and a close idea of reason which does not believe in the possibility of discovering truth into experience.

KeY words: Galileo, Descartes, science, method, reason, modernism, mechanicalism

\section{LA LEYENDA DE LOS DOS PADRES DE LA CIENCIA}

Cegún una opinión muy común, compartida por casi todos los historiadores y los filósofos, de modo que se encuentra en casi todos los libros para la escuela (y, lamentablemente, también en muchos de nivel más alto), la ciencia moderna habría sido inventada por Galileo Galilei y René Descartes. Esta idea no es solo errónea, sino que tiene profundas consecuencias culturales, pues a partir de ello deriva que el racionalismo y el mecanicismo modernos — que nacieron precisamente por obra de Descartes - sean partes esenciales de la ciencia como tal. Al contrario, las cosas son muy diferentes, ya que si examinamos los hechos objetivamente, podemos ver facilmente que el verdadero padre de la ciencia fue Galileo, y únicamente Galileo; mientras que Descartes fue más bien el padre del cientificismo (es decir, de la transformación de la ciencia en ideología 
materialista, a pesar de que esta no era su intención), y no tiene nada que ver con la ciencia real, pues su famoso «método» es exactamente opuesto a el de Galileo.

\section{GALILEO Y EL ORIGEN DE LA CIENCIA}

Cuando Galileo empezó su trabajo, algunos descubrimientos científicos importantes ya se habían dado, sobre todo en matemática (gracias al redescubrimiento de los textos de los griegos antiguos que fueron transmitidos por los árabes), pero también algunos en el campo de las ciencias de la naturaleza. En particular, el polaco Copernico (Nicolas Koppernigk, 14731543) ya en 1543 había editado su libro fundamental De revolutionibus orbium coelestium, en el que proponía su teoría heliocéntrica (es decir, con el Sol en el centro y la Tierra y demás planetas que giran alrededor de él), que se oponía a la geocéntrica (con la Tierra en el centro y todos los demás que giran alrededor de ella), que entonces era la generalmente aceptada. Además, en el mismo 1543, el belga Andrea Vesalio (Andreas Van Wesel, 1516-1564) había empezado los primeros estudios sistemáticos de anatomía humana; en 1569, el holandés Gerardo Mercatore (Gerard De Cremer, 1512-1594) había creado el primer mapa geográfico moderno (tan moderno que su método, la "proyección de Mercatore», se usa todavía hoy); entre el 1576 y el 1597, el danés Tycho Brahe (1546-1601) había realizado las primeras observaciones astronómicas precisas y sistematicas; en 1582, el jesuita Christophorus Clavius (1537-1612), el más grande matemático de su tiempo y gran amigo de Galileo, había llevado a cabo la reforma del calendario gregoriano (tan preciso que sigue siendo todavía utilizado hasta nuestros días); en 1583, el italiano Andrea Cesalpino (1519-1603) había dado la primera clasificación coherente de las plantas; y, por fin, en 
1586 el belga Simon Stevin (1548-1620) había descubierto las leyes de la hidrostática. Entonces, ¿̨por qué decimos que la ciencia moderna comienza con Galileo?

En este tipo de discursos siempre hay algo convencional, porque ninguno crea nada de la nada, y siempre hay, para así decirlo, un trabajo de equipo, que nace dentro de una tradición. Pero Galileo indudablemente por primera vez realizó lo siguiente:

1) Probó la unidad de la naturaleza (es decir, que las mismas leyes son válidas tanto en la Tierra como en las estrellas más distantes), gracias a sus descubrimientos astronómicos.

2) Probó las primeras leyes naturales realmente fundamentales, las del movimiento de los cuerpos (que, por lo que hemos dicho en el punto anterior, no eran validas solamente para la Tierra, sino para todo el Universo).

3) Estableció de una manera clara y definitiva el método de la ciencia natural.

Ahora bien, cada una de estas contribuciones ya tiene una importancia trascendental y sería suficiente para asegurarle a Galileo un lugar de primer nivel en la historia de la ciencia. Por lo tanto, se puede entender facilmente por qué, realizando las tres en conjunto, Galileo se haya realmente convertido en el padre de la moderna ciencia natural, como ahora vamos a ver un poquito más de cerca.

\section{I. LOS DESCUBRIMIENTOS ASTRONÓMICOS Y LA UNIDAD DE LA} NATURALEZA

Como desde hace tiempo ha sido aclarado por los historiadores, el telescopio no fue inventado por Galileo, sino por el holandés Hans Lippershey (1570- 
1619), en el 1608. Pero Galileo, que nunca lo vio, sino que solamente escuchó hablar de eso por su amigo Paolo Sarpi (1552-1623), entendió perfectamente su principio básico (aunque sin llegar a una teoría formal da la óptica, lo que hizo Kepler en el 1611), hasta el punto que no solo fue en grado de reconstruirlo, sino también de perfeccionarlo muchísimo. Pero sobre todo Galileo tuvo la idea de aprovecharlo para estudiar el cielo, mientras que hasta entonces únicamente lo habían usado para observar la tierra: lo que demuestra cómo a veces es suficiente usar un instrumento de una manera nueva para convertirlo, en un sentido, en un nuevo instrumento; y que por tanto no existe una observación "puramente empírica», como muchas veces se dice, pues siempre la observación tiene que basarse en alguna idea, como veremos mejor más delante. Así Galileo, en menos de tres años, descubrió: las montañas lunares; el fenomeno de la llamada «luz de cineza» (es decir, la débil luz que ilumina la parte oscura de la Luna, que es la luz del Sol que rebota de la Tierra en la Luna); la diferencia entre planetas y estrella (pues solamente los primeros se ven engrandecidos por el telescopio); el inmenso número de estrellas (mucho más grande de lo que se puede ver a simple vista); la verdadera naturaleza de la Vía Láctea (formada también de estrellas); los cuatro mayores satélites de Júpiter; los anillos de Saturno (aunque sin darse cuenta de lo que eran realmente); las fases de Venus (que le dieron la prueba final en contra del sistema tolemaico); y, por fin, las manchas del Sol. De esta manera, demostró que los cuerpos celestes no son esferas perfectas ni tampoco son hechos de una materia especial, como se creía, y por tanto no son una realidad aparte, sino que son de la misma naturaleza de la Tierra. Además, la existencia de un centro de rotación diferente de la Tierra, o sea Júpiter, quitaba otra importante justificación al geocentrismo. Por fin, las fases de Venus demostraban indudablemente la falsedad del sistema tolemaico, del cual no se podían 
absolutamente deducir, aunque esto aún no era suficiente para demostrar la verdad del sistema heliocéntrico, pues ellas se podían explicar en el marco del sistema tychonico, que seguía siendo geocéntrico, pues en eso los planetas sí giraban alrededor del Sol, pero el Sol todavía alrededor de la Tierra.

\subsection{LAS LEYES DEL MOVIMIENTO Y LA DEFENSA DEL HELIOCENTRISMO}

Además, Galileo descubrió las leyes del movimiento de los cuerpos, que, demás de su importancia intrínseca, probaron que el sistema copernicano podía ser real, aunque no que era real de hecho: a Galileo para demostrar definitivamente la teoría heliocéntrica siempre le faltó la prueba final, la del paralaje de las estrellas, que solo llegó en 1837 por obra de Friedrich Wilhelm Bessel (1784-1846). El heliocentrismo, en efecto, no era una novedad: ya lo había propuesto en la antigua Grecia Aristarco de Samo (310-230 a.C.). Pero había un problema: ¿por qué los cuerpos caen verticalmente? En efecto, si la Tierra se mueve debajo de los cuerpos mientras estos caen, deberían llegar a la superficie terrestre ligeramente desplazados de la perpendicular y no verticalmente como aparentemente ocurre. La objeción parece muy natural, pero esto es lo mismo que pasa con una nave: por tanto, habría sido suficiente hacer el experimento para descubrir la verdad. Pero, increíblemente, el experimento era tan simple que ninguno lo había hecho realmente, puesto que todos creían que ya otros de seguro lo habrían hecho antes que ellos. Galileo, en cambio, ya en 1613, en Las manchas del Sol, había entendido que las cosas no son así, y en 1632, en sus famosos Diálogos sobre los dos máximos sistemas del mundo, tolemaico y copernicano, logró demostrarlo, gracias a un famoso experimento mental:

Salviati. Me parece que hasta ahora usted me ha explicado lo que le pasa a un cuerpo en movimiento sobre dos diferentes planos, y 
que en el plano inclinado el cuerpo baja espontáneamente y sigue acelerándose continuamente, y que para hacer que permanezca inmóvil es necesario retenerlo con la fuerza; pero en el plano ascendente es necesaria la fuerza para empujarlo y también para retenerlo, y que el movimiento que hemos dado a ese cuerpo sigue disminuyendo continuamente, así que finalmente termina. [...] Y ahora dígame qué pasaría al mismo cuerpo sobre una superficie que no fuese ni ascendente ni descendente. [...] Simplicio. Yo no puedo ver alguna causa de aceleración ni de desaceleración, pues que no hay ni declinación ni ascensión. Salviati. Sí. Pero si no fuese alguna causa de desaceleración, aún menos debería ser causa de inmovilidad: y por lo tanto, según usted, ¿̨hasta cuándo el cuerpo debería seguir moviéndose? Simplicio. Hasta cuando siga prolongándose aquella superficie ni ascendente ni descendente. Salviati. Por lo tanto, si dicho espacio fuese infinito, el movimiento en ese espacio sería de la misma manera infinito, es decir perpetuo. (Galilei 1632: 172-173) (1879-1955), que no tenía solo (obviamente) una profunda comprensión de 
la física, sino también de su historia, ${ }^{1}$ nunca tuvo dudas a este propósito. Por ejemplo (pero que es realmente solo un ejemplo entre muchos que se podrían hacer), en Mi visión del mundo, hablando de Galileo, dice claramente que «fue él que descubrió la ley de la inercia» (Einstein 1934: 85). Y fue exactamente gracias a este descubrimiento que Galileo pudo eliminar todas las objeciones en contra del sistema heliocéntrico, y así empezó la primera revolución cosmológica de la historia. Pero en Galileo hay mucho más que únicamente el principio de la inercia. En efecto, él descubrió también la relatividad del movimiento, que nunca puede ser definido en absoluto, sino solo relativamente a un sistema de referencia (o sea, otro cuerpo u otro conjunto de cuerpos), aunque la relatividad galileana se refería exclusivamente a los sistemas de referencia inerciales, es decir, a los que tienen un movimiento rectilíneo uniforme. La relatividad de Einstein, como él mismo siempre reconoció lealmente, es simplemente la extensión del principio galileano a los fenómenos electromagnéticos (que Galileo no conocía) y a los sistemas no inerciales, es decir, acelerados (aunque esto, por la particular naturaleza de la luz, necesitó un profundo cambio en nuestra concepción del espacio y del tiempo: pero esta es otra historia). Por fin, Galileo llevó a cabo su trabajo acerca de las leyes del movimiento de los cuerpos demostrando, con otro famoso experimento mental, que los cuerpos caen todos con la misma velocidad y no con velocidad proporcional a su peso, como decía la física aristotélica, así abriendo el camino hacia el reconocimiento de la identidad de la masa inercial con la gravitacional y, por consiguiente, a la teoría de la relatividad general (pero que, de nuevo, es otra historia).

1 En efecto, Einstein, junto al polaco Leopold Infeld (1898-1968), escribió también uno de los textos fundamentales en materia, La evolución de la física, que en mi opinión sigue siendo el mejor de todos, a pesar de sus 73 años, por su capacidad de juntar, de una manera incomparable, la profundidad con la sencillez. 


\subsection{El MÉtodo de LA CIENCIA NATURAL}

Además de sus descubrimientos, Galileo estableció también el método de la ciencia natural, que, en un sentido, fue hasta más importante, pues permitió todos los descubrimientos posteriores, hasta nuestros días y más allá, pues su método sigue siendo valido después de cuatro siglos, que, si pensamos bien en esto, es increible. Pero esto no solamente fue un logro práctico, sino, como bien entendió de nuevo Einstein, «uno de los más importantes acontecimientos en la historia del pensamiento humano» (Einstein e Infeld 1938: 19), pues fue nada menos que el descubrimiento de un nuevo modo de usar la razón; lo que, como se entiende fácilmente, no es algo que acontece todos los días. Y estos son, en orden de importancia, los cuatro principios básicos del método científico según Galileo:

1) No buscar la esencia, sino limitarse a estudiar algunas propiedades.

2) No solamente una genérica observación, sino experimentos, que se hacen en condiciones artificiales, orientadas y repetibles.

3) Demostraciones matemáticas.

4) Ningún principio de autoridad.

Aunque obviamente todos los cuatro principios tienen que estar juntos para que el método pueda funcionar, el más importante es el primero. En efecto, desde la Grecia antigua hasta Galileo, todos siempre intentaron hacer la ciencia natural a priori, es decir, según el método deductivo, que busca en primer lugar establecer los principios más fundamentales y generales y después deducir de ellos todos los fenómenos particulares, lo que tuvo mucho éxito en metafísica, lógica, matemática y especialmente en geometría con los Elementos de Euclídes (siglo iv-283 a.C.), un texto tan moderno que fue mejorado por primera vez en su estructura básica por el alemán David 
Hilbert (1862-1943) en 1899, es decir, ¡después de más de 22 siglos! Por tanto, en un sentido, los antiguos fueron víctimas de sus primeros éxitos, pues éstos los empujaron muy naturalmente a pensar que dicho método fuese valido por todo conocimiento.

Galileo, en cambio, entendió primero que el método del conocimiento humano no tiene que ser necesariamente único y que en el caso de la ciencia de la naturaleza (y solo en este caso) era necesario invertir el método, empezando en cambio por los aspectos más simples y particulares, para después llegar a los más profundos y generales. Esto se aprecia sobre todo en el famoso texto de Las manchas del Sol, donde encontramos su primera y más famosa formulación:

Por lo tanto, o nosotros queremos especular intentando penetrar la esencia verdadera e intrínseca de las sustancias naturales; o nosotros queremos contentarnos en descubrir algunas de sus propiedades. Buscar la esencia, yo creo que es un intento tan inútil en el caso de las sustancias muy simples como en lo de las más lejanos y celestes: y me parece que soy igualmente ignorante sobre la sustancia de la Tierra que de la Luna, de las nubles y de las manchas del Sol; ni veo que comprendiendo estas sustancias cercanas tenemos otra ventaja que el número de las propiedades, pero todas igualmente desconocidas. [...] Pero si queremos limitarnos en aprender algunas propiedades, no me parece que sea imposible descubrirlas también en los cuerpos más lejanos de nosotros, no menos que en los cercanos. (Galilei 1613: 187-188) 
Muchos historiadores y epistemólogos no están de acuerdo con mi interpretación, ${ }^{2}$ ya que eligen a uno de los otros principios como el más importante. Sin embargo, se puede ver fácilmente que hay una estrecha relación lógica entre los cuatro principios galileanos, que son concatenados uno tras otro, de modo tal que los otros tres dependen todos, directamente $\mathrm{o}$ indirectamente, del primero.

En primer lugar, en efecto, el experimento se diferencia de la simple observación porque es algo artificial, orientado y repetible. Pero esto solo es posible exactamente porque tiene por tarea evidenciar y estudiar «algunas propiedades», y por tanto se funda en el primer principio: si no fuese así, nunca sería posible cumplir con dichas condiciones, pues ningún fenómeno nunca se repite perfectamente igual en todos sus factores. Además, esto significa que el experimento siempre implica una hipótesis teórica de algún tipo, sin la cual ni siquiera podría ser concebido, pues sin una hipótesis qualquiera no es posible establecer cúales propiedades el experimento tiene que evidenciar. Por esto, en la ciencia hablar de mero empirismo o de materialismo no tiene ningún sentido, aunque esto no significa tampoco que el experimento pueda reducirse a la teoría, como sugiere el antirrealismo epistemológico contemporáneo. En efecto, ambos aspectos, el teórico y el empírico, son esenciales, y siempre tienen que estar juntos para que la ciencia pueda funcionar.

En segundo lugar, también la matemática, para poder aplicarse al estudio de la naturaleza, tiene necesidad de un objeto definido de manera precisa y no ambigüa: por ejemplo, las leyes del movimiento solamente describen la trayectoria de un cuerpo y no su color o su sabor. Luego,

2 Que, de todos modos, no es únicamente mía, pues ha sido propuesta por catedráticos ilustres, como por ejemplo Evandro Agazzi (1934-vivo), Stanley Jaki (1924-2009), Peter Hodgson (1928-2008) y Sofia Vanni Rovighi (1908-1990). 
también este principio para funcionar tiene necesidad de limitarse al estudio de «algunas propiedades», y por lo tanto presupone el primero.

Finalmente, Galileo sí rechaza el principio de autoridad, pero solo porque en la ciencia hay una autoridad superior, la de la naturaleza (que ultimamente es la de Dios mismo, que es su Creator y nos habla a través de esta), que todos pueden interrogar por medio de la matemática y del experimento. ${ }^{3}$ Pues, como hemos visto, el uso de la matemática y del experimento se funda a su vez en el primer principio, entonces también este cuarto principio depende de eso. Por consiguiente, el primer principo «no buscar la esencia», es realmente el principio fundamental del método de Galileo.

Hay que subrayar que, como Galileo dice claramente en el texto que hemos citado antes, todo esto vale solo en el caso «de las sustancias naturales», o sea, de los «cuerpos». Es decir, para Galileo el método depende del objeto: no hay un método único del conocimiento que va bien para todo, sino, por el contrario, objetos diferentes piden métodos diferentes (pluralismo metodólogico, no reduccionismo). Esto también fue un increible progreso en el modo de concebir la razón, aunque hoy pueda parecer normal. En efecto no lo era, porque Galileo compartía con la gran mayoría de sus contemporáneos el ideal (en sí mismo bueno) de la unidad del saber, que parecía implicar la existencia de un único método para lograr un verdadero conocimiento acerca de cualquier argumento. Por tanto, fue realmente una intuición genial la de Galileo que esta idea podía ser engañosa y que en el

3 Véase por ejemplo este texto: «Pues proceden igualmente del Verbo divino la Sagrada Escritura y la naturaleza, aquella como dictada del Espíritu Santo, y esta como observantísima ejecutora de las ordenes de Dios; [...] parece que aquello entre los efectos naturales, que, o la experiencia sensible nos pone delantede los ojos o las necesarias demostraciones nos hacen concluirlo, no se pueda dudar por ninguna razón» (Galilei 1615: 282). 
campo de la ciencia natural podía ser necesario un cambio metodológico. En esto, Galileo fue probablemente ayudado por su fe cristiana en la Creación, ya que esta implica que el mundo no es un ser necesario, sino contingente, o sea, el mundo es como es porque Dios así lo quiere, pero habría también podido ser diferente. En efecto, si el mundo es contingente no hay principios necesarios de la naturaleza que se puedan buscar solo con la razón pura, como en lógica, matemática o metafísica, y por tanto, es necesario empezar de los aspectos particulares. Como he dicho, personalmente creo que esto tuvo su importancia también desde un punto de vista historico en la revolución galileana, pues ha sido demostrado de una manera convencida que esta fue preparada por un largo proceso de critica de la fisica aristotelice que empezó exactamente después de que el día 7 de marzo del 1277 el Obispo de Paris Étienne Tempier (?-1279) había condenado como herética, entre muchas otras, la tesis averroista "Quod Prima Causa non posset plura munda facere» («Que la Primera Causa [es decir, Dios] no pueda crear muchos mundos diferentes»), que negaba la omnipotencia divina (Cf. Musso 2011: 76-84). De todos modos, indipendientemente de este problema, desde un punto de vista objetivo ésto es el fundamento metafísico último de la inversión metodologica galileana.

Además, es importante entender que esta autolimitación al estudio de las propiedades solo es metodológica. Galileo no era un fenomenista ni mucho menos un escéptico: por el contrario, en toda su vida él siempre dijo de una manera extremadamente clara que su investigación era acerca del verdadero orden del mundo, y exactamente por esto fue procesado, lo que nunca habría acontecido si hubiera aceptado el consejo del cardenal Roberto Bellarmino (1542-1621) de «hablar ex suppositione» (Bellarmino 1615; 171), es decir, presentando sus ideas como meras hipótesis matemáticas. Por ende, Galileo no rechaza la investigación de la esencia de las cosas (que 
a su tiempo significaba simplemente «lo que las cosas son realmente»), ${ }^{4}$ pero en su método esta se convierte en el punto de llegada de la investigación científica (que se puede lograr solo por grados e imperfectamente) en lugar de ser su punto de partida, como era para los antiguos.

Finalmente, debemos añadir que todo esto vale en principio, mientras que es claro que en la práctica, si cada científico tuviera que volver cada vez a empezar todo desde el principio, el progreso científico sería imposible. Por consiguiente, de hecho, ni siquiera la ciencia puede prescindir completamente del método del conocimiento por fe (que usamos además en la gran mayoría de nuestra vida diaria) y luego ni mucho menos del principio de autoridad. Por tanto, hay una tensión entre el aspecto teórico y lo práctico de la ciencia, que es inevitable y que cada científico tiene que manejar lo mejor que pueda, intentando entender cuándo es oportuno confiar con los ojos cerrados y cuándo, en cambio, es mejor controlar personalmente cada paso. En esto juega un papel importante no solo la experiencia profesional, sino también la humana, porque en la elección de confiar no entra en juego solamente un juicio técnico sobre la preparación científica del otro, sino también un juicio sobre su fiabilidad como persona (método de la certeza moral). Por tanto, tampoco el método científico, por más objetivo e impersonal que sea, puede prescindir completamente del factor humano, aunque el hecho de que en la ciencia, en principio, siempre podemos controlar cualquier afirmación es de seguro un factor extremadamente importante y que la hace diferente de todas las otras formas de conocimiento humano.

4 Fue solo con Immanuel Kant (1724-1804) que el término esencia se retomó para indicar algo misterioso e inconocible que está «más allá» de los fénomenos. Por tanto la tesis de un Galileo fenomenista no solo no es correcta, sino que no puede ser correcta, pues se basa en un claro anacronismo. 


\section{DESCARTES Y EL MECANICISMO MODERNO}

Como todos saben, el francés René Descartes (1596-1650) fue el que comenzó el mecanicismo filosófico $0^{5}$ moderno, que, por algún tiempo, pareció ser confirmado también por los descubrimientos científicos, hasta convertirse, para así decirlo, en la visión «oficial» del mundo durante los siglos XVIII y XIX.

Cuando se habla de mecanicismo, la imagen que se genera espontaneamente en nuestra mente es la de partículas inmutables (los átomos) que se juntan y se separan en un espacio vacío: pero, sorprendentemente, Descartes no admitía ni las primeras ni el segundo. ${ }^{6} \mathrm{Y}$ por tanto, ¿qué se entiende exactamente por "mecanicismo»? Quizás la mejor manera de explicarlo es por contraposición a la filosofía clásica, que admitía cuatro tipos de mutación:

1) sustancial (generación y corrupción);

2) cualitativa (alteración);

3) cuantitativa (crecimiento y disminución);

4) local (movimiento).

Por lo contrario, para Descartes «toda diversidad de las formas [...] depende del movimiento local» (Descartes 1644: 81). O sea, la esencia del mecanicismo está en el hecho de que todo cambio se explica por el

5 Digo «filosófico» porque el mecanicismo científico, aunque a veces pueda parecer la misma cosa, en efecto, como veremos, es algo muy diferent

6 La razón es que, pues, como veremos en lo que sigue, Descartes identificaba la materia con la pura extensión geométrica, claramente de esto derivaba que no pueden existir ni un espacio vacío (pues tenenedo una extensión tiene que ser materia) ni partículas indivisibles (pues cualquier forma geométrica extensa siempre se puede dividir ulteriormente, ya que los constituenes fundamentales del espacio geométrico son puntos sin dimensiones, para llegar a los cuales sería necesario un número infinito de divisiones). 
movimiento. $\mathrm{Y}$ en efecto, a pesar de que en sus obras haya dado varias listas de reglas metodológicas, que van de las 4 del Discurso sobre el método (Cf. Descartes 1637a: 303) a las 21 de las Reglas para la dirección de la inteligencia (Cf. Descartes 1628), el famoso «método» de Descartes básicamente se reduce a dos pasos fundamentales, que corresponden fundamentalmente a la segunda y a la tercera regla del Discurso:

1) análisis: se va a descomponer el objeto que se quiere estudiar en sus partes simples; ${ }^{7}$

2) sintesis: se van a juntar de nuevo todas esas partes simples según sus leyes, ${ }^{8}$ que en el caso de los cuerpos fisicos son las leyes de su movimiento.

Por consiguiente, los seres complejos (incluidos nosotros) no tienen una auténtica individualidad, pues no son nada más que simples agregados de partes: y en efecto, como es bien notorio, para Descartes todos los seres vivos deben ser considerados máquinas, con la única excepción del hombre, pero solo porque Descartes lo identifica con su alma, mientras que su cuerpo es también una máquina.

Además, obviamente el hecho de que cualquier objeto complejo en principio pueda ser descompuesto en sus partes simples, lleva a la inevitable consecuencia que la ciencia — por lo menos en principio — pueda contestar a cualquier pregunta, como lo dijo de la manera más clara y celebre, en 1776, en su famoso Essai philosophique sur les probabilités, Pierre-Simon de Laplace (1749-1827):

7 «Dividir cada una de las dificultades que estaba examinando en el mayor número posible de partes, al fin de una mejor solución» (Descartes 1637a: 303).

8 «Imponer un orden a mis pensamientos, empezando por los objetos más simples y faciles para conocer, y luego remontar un poquito a la vez, como por grados, hacia el conocimiento de los más complejos» (Descartes 1637a: 303). 
Tenemos por tanto que estimar el presente estado del Universo como el efecto de su estado anterior y como la causa de su estado futuro. Una Inteligencia que, en un determinado momento, conocería todas las fuerzas por las que es animada la naturaleza y toda la situación respectiva de los seres que la componen, si además fuese bastante profunda como para someter dichos datos al análisis, abrazaría en la misma fórmula los movimientos de los cuerpos más grandes del Universo y del átomo más ligero: nada sería incierto para esa Inteligencia y el futuro, como el pasado, sería presente a sus ojos. El espíritu humano, en la perfección que ha podido dar a la astronomía, es un pálido ejemplo de esta Inteligencia. Sus descubrimientos en la mecánica y en la geometría, juntos a aquello de la gravitación universal, lo han hecho capaz de abrazar en las mismas expresiones analíticas los estados pasados y los futuros del sistema del mundo. (Laplace 1776: 243) ${ }^{9}$

Por tanto, como se ve, la concepción cartesiana lleva inevitablemente hacia la idea de la omnipotencia de la ciencia, es decir, hacia el cientificismo. Y, en efecto, Descartes mismo no solo estaba convencido de que gracias a su método se podían explicar todos los fenómenos de la naturaleza, sino que ya había resuelto «cinco o seis problemas principales» (Descartes 1637a: 335) y que solo le hacía falta "ganar otras dos o tres [batallas] de la misma importancia para llevar completamente a cabo mis proyectos» (Descartes 1637a: 335). Sin embargo, para Descartes su método

9 Aunque hay que decir que Laplace de inmediato seguía reconociendo que «todos sus esfuerzos en la búsqueda de la verdad tienden a acercarlo continuamente a la Inteligencia que hemos imaginado, pero de la que se quedará siempre infinitamente lejos» (Laplace 1776: 243). 
PAOLO MUSSO

era en primer lugar el método de la filosofía, que después sí pasaba a ser también el método de la ciencia, pero solo después.

\section{I. UN PENSADOR PREGALILEANO}

La razón es que Descartes todavía tenía una concepción pregalileana de la ciencia natural, que para él tenía que ser deducida por los principios de la filosofía, como él mismo dice explicitamente: «habendome dado cuenta que los principios científicos debían todos depender de la filosofia, pensé que, en primer lugar, tenía que intentar establecer en esta algunos principios ciertos que aún no podía encontrar»(Descartes 1637a: 305). En efecto, a pesar de que sea generalmente presentado como un valiente científico e muchas veces hasta como el que comenzó la ciencia moderna junto a Galileo, la realidad es que Descartes malentendió completamente la novedad del método galileano. Él critica a Galileo porque «busca las razones de algunos efectos particulares y no toma en consideración las causas primeras de la naturaleza» (Descartes 1638: 387), hasta el punto de sostener que en sus libros «no hay practicamente nada que querría haber escrito yo» (Descartes 1638: 391); dice que "toda la filosofía es como un árbol, cuyas raíces son la metafísica, el tronco es la física y las ramas que del tronco provienen son todas las otras ciencias» (Descartes 1644: 15) e incluso que de un principio meafísico como el de la «inmutabilidad de Dios, y del hecho que siempre actúa de una misma manera, podemos llegar al conocimiento de algunas reglas, que yo llamo leyes de la naturaleza» (Descartes 1644: 91); y, por fin, que «quien sepa examinar suficientemente las consecuencias de tales verdades y de nuestras reglas podrá conocer los efectos a partir de las causas y, para usar los términos de la Escuela, podrá tener demostraciones a priori 
de todo lo que puede ser producto en este nuevo10 mundo» (Descartes 1664: 154).

En otras palabras, para Descartes el método de la ciencia natural sigue siendo puramente deductivo, pues empieza por los principios primeros (es decir, metafísicos), que él piensa se puedan alcanzar por el puro pensamiento (lo que Galileo llamaba «buscar la esencia»), y después intenta deducir de esos todas las propiedades particulares, exactamente como era para los antiguos, y especialmente para los filósofos aristotélicos que a palabras Descartes critica, pero sin darse cuenta de que, aunque sus principios sean diferentes, el marco general en que los coloca todavía sigue siendo esencialmente igual.

Además, él habla de experimentos, pero en sus ejemplos se ve claramente que en realidad se refiere a simples observaciones (que muchas veces ni siquiera hizo personalmente, y muy a menudo son erróneas). Sí, usa la matemática, pero como modelo de su método deductivo (como los antiguos), y no como instrumento (como en cambio hizo Galileo). Asimismo, rechaza la autoridad, pero no porque piensa (como Galileo) que todos puedan usar su método para establecer quien tiene razón, sino porque piensa que solo él puede realmente entenderlo. Por consiguiente, Descartes llega a negar también las dos principales características de la ciencia moderna fundada por Galileo: la de ser por su naturaleza un trabajo común; y la de ser por su naturaleza un trabajo abierto, en continuo progreso y en continua evolución.

Todo esto no vale solo desde un punto de vista teórico, sino que es confirmado también por la historia de la ciencia. De hecho, Descartes no dio ninguna contribución directa a la ciencia natural, aunque dio algunos aportes

10 Acá Descartes dice «nuevo» porque en El Mundo adopta el espediente retórico de decir que solo esta hablando de la creación de un nuevo mundo imaginario. Pero no hay duda que en efecto quiere hablar del mundo real. 
PAOLO MUSSO

fundamentales (pero indirectos) con sus descubrimientos matemáticos, especialmente el de la geometría analítica. En efecto, su famosa «física de los torbellinos» fue construida completamente a priori, se reveló no solo errónea, sino contradictoria (porque Descartes no admitía la existencia del vacío, pero el movimiento de los torbellinos implica que entre las partículas se formen necesariamente espacios vacíos) y, sobre todo, no contenía ni una formula matemática. En efecto, cuando Isaac Newton (1642-1727) quizo demostrar su falsedad, tuvo que traducirla toda en términos matemáticos por su cuenta; y cuando lo hizo, descubrió que el orden de magnitud del error de las predicciones acerca del movimiento de los planetas que derivaban de la teoría cartesiana era más de 1000 veces mayor del orden de magnitud del error de las mejores observaciones de entonces (Cf. Banfi 2007): lo que prueba definitivamente que Descartes nunca intentó verificar su teoría experimantalmente, ni siquiera aproximadamente, pues de seguro no era posible equivocarse de ninguna manera acerca de un error de esta magnitud.

Tampoco descubrió ni el principio de la inercia ni el de acción y reacción. Es verdad que Descartes también tuvo una intuición del principio de la inercia, que enunció más tarde que Galileo, ${ }^{11}$ pero en cambio más claramente, pues él lo refiere solo al movimiento rectilíneo uniforme, mientras que Galileo, como hemos visto, pensaba, erróneamente, que se aplique también al movimiento circular uniforme. Sin embargo, en Descartes se queda así: una intuición, y nada más. Y exactamente aquí está toda diferencia, a no decir el abismo, entre la concepción de la ciencia típica tanto

11 En efecto, Descartes lo enunció por primera vez en El Mundo, que llevó a cambo en 1633, pero nunca publicó (salió póstumo solo en 1677), hizo referencia a eso (pero sin enunciarlo explicitamente) en el Discurso sobre el método (1637) y lo publicó por primera vez en Los principios de la filosofía solo en 1644. Galileo en cambio dio su demostración, de que hemos hablado antes, en su celebre Diálogos sobre los máximos sistemas (1632), pero ya lo había enunciado en 1613 en Las manchas del Sol. 
de Descartes como de los antiguos, que pretendían basarse exclusivamente en una intuición intelectual de las propiedades fundamentales de la naturaleza (las «esencias»), y el método galileano, que en cambio nos obliga a basarnos constantemente en las «experiencias sensibles» y las «necesarias demostraciones» (Galilei 1938: 212). Tal es la potencia de este método, que, siguiéndolo, incluso se puede llegar a convertir una intuición todavía imperfecta, como la que tuvo Galileo, en uno de los pilares de la ciencia de los siglos futuros; mientras que, en cambio, siguiendo el otro camino aún la intuición más feliz y exacta se encuentra condenada a una inescapable esterilidad.

Esto se ve muy bien en la física de Descartes, en la cual la falta de cualquier control experimental hace que dicho principio sea usado para edificar una construcción de pura fantasía, como hemos visto en lo anterior, y sobre todo en lo que él dice acerca del principio de acción y reacción, que en efecto solo impropiamente se puede llamar así, pues en Descartes ni siquiera es un único principio, sino más bien se encuentra enunciado de 7 diferentes maneras en 7 diferentes leyes, que según él se refieren a 7 diferentes situaciones (Cf. Descartes 1644: 97-102), que en cambio el auténtico principio de acción y reacción, enunciado por Newton en 1687 (Cf. Newton 1687), los trata todos de una manera unitaria. Además, todas estas 7 formulaciones solo son mecánicas (mientras que el principio de Newton vale por cualquier tipo de interacción, empezando por la gravedad, que no es mecánica pues actua a distancia y no por contacto) y todas son incorrectas. Lo más grave, sin embargo, es que en estas se ve muy claramente que Descartes, a pesar de su correcta intuición del principio de la inercia, no había entendido bien su sentido profundo y, sobre todo, sus consecuencias, en primer lugar la relatividad del movimiento. En efecto, introduciendo el tema él dice que «solo hay contrariedad en dos modos. Es decir, entre el movimiento y la 
PAOLO MUSSO

quietud, o más bien entre la velocidad y la lenteza del movimiento, pues dicha lenteza participa en la naturaleza de la quietud» (Descartes 1644: 96), y poco más delante se ve muy claramente, del modo en que explica sus 7 leyes, que este texto tiene que ser interpretado literalmente, es decir, en el sentido de que Descartes piensa realmente que hay una diferencia de naturaleza entre el movimiento y la quietud. Por razones de espacio, voy a hacer solo el ejemplo más clamoroso, donde afirma: «La cuarta [ley] es que, si el cuerpo C fuese más grande que $\mathrm{B}$, hasta poco, y fuese enteramente en quietud, [...] con cualquier velocidad B pudiese venir en contra de ello, nunca habría la fuerza para moverlo, sino que sería obligado a rebotar hacia el mismo lado donde había venido» (Descartes 1644: 99). Acá no solo se encuentra, de la manera más clara, la prueba de lo que hemos dicho en lo anterior, sino que se hace evidente también la naturaleza completamente a priori de la manera de razonar de Descartes. En efecto, lo que dice está completamente en contra de la experiencia: por tanto, parece natural preguntarse cómo sea posible que él no se dea cuenta. Pero no tenemos que hacer ningún esfuerzo para buscar la solución de esta paradoja, pues el mismo Descartes nos la explica 3 páginas adelante, donde afirma que «las demostraciones de todo esto son así ciertas, que incluso si la experiencia parecería hacernos ver lo contrario, no obstante deberíamos creer más a nuestra razón que a nuestros sentidos» (Descartes 1644: 102). Una vez más, por tanto, se ve aquí como el único método que Descartes ha conocido fue siempre y solo el deductivo, al igual que los filósofos aristotélicos. Y en efecto sus tratados «físiscos» (Los principios de la filosofía, El Mundo y El Hombre) tienen un «sabor», por así decirlo, muy parecido a los de la filosofía escolástica de la decadencia ${ }^{12}$ que en principio él tanto desechaba: y no acaso, pues, como ya hemos dicho y

12 Para una colección de ejemplos a este propósito véase Musso 2011: 188-192. 
como lo anterior confirma, solo el punto de partida era diferente, mientras que el método se quedaba idéntico.

Por fin, hay que decir que Descartes no hizo ningún descubrimiento ni siquiera en el campo de la óptica geométrica, a pesar de que le haya dado una formulación matemática moderna (Cf. Descartes 1637b). Pero las leyes fundamentales ya habían sido todas descubiertas: la de la refracción de la luz en 1621 por el holandés Willebrord Snell Van Royen (1580-1626) y las otras hasta más antes por Johannes Kepler (1571-1630), que en 1611 le recogió todas en su Dioptrice (Cf. Kepler 1611). Además, su propuesta de usar lentes hiperbólicas en los telescopios era errónea y su máquina para fabricarlas (a pesar de los continuos cambios que aportó al proyecto) simplemente no funcionaba. El mundo de la ciencia experimental era tan ajeno para Descartes que ni siquiera logró traducir en términos físicos correctos su única intuición realmente nueva, o sea, que el arco iris es causado por la refracción de la luz solar en las pequeñas gotitas de agua que permanecen suspendidas en el aire después de la lluvia, analogamente a lo que acontece (como ya se sabía en la Edad Media) cuando la luz pasa a través de un prisma de vidrio.

Por tanto, en conclusión, la verdad es que Descartes fue un gran filósofo y un gran matemático, ${ }^{13}$ pero nunca fue un cientifico en el sentido moderno del término: es más, fue, en todo sentido, un pensador pregalileano. Por consiguiente, tampoco su mecanicismo no tiene ninguna relación con la ciencia, sino nace en un ámbito muy diverso.

$13 \mathrm{Y}$, esto sí, como matemático fue hasta más grande de lo que generalmente se piensa, pues sin la geometría analítica nunca habría sido posible hacer geometría en mas de 3 dimensiones, como perfectamente entendió de nuevo Einstein (Cf. Einstein 1934: 124126). Pero esta es otra historia. 
PAOLO MUSSO

\subsection{El DOgMA CENTRAL DE LA MODERNIDAD}

Como hemos dicho, el primer objetivo de Descartes era establecer un método eficaz para la filosofía, lo que él creía posible lograr imitando el de la matemática. Por ello, en primer lugar era necesario encontrar un «comienzo» absoluto, es decir, una verdad tan evidente que no sea posible dudarla de ninguna manera, como los axiomas de la geometría o hasta más. Como todos sabemos, este «comienzo» de la filosofía Descartes creyó encontrarlo en el famoso «Cogito ergo sum» ('Pienso luego existo'; Descartes 1637a: 312), porque esta es la única cosa que resiste al test de la duda metodológica, o sea, es la única cosa que sigue siendo necesariamente verdadera incluso si todo lo que veo y lo que pienso fuese falso, por ejemplo, por qué hay un diablo muy poderoso que me engaña. ${ }^{14}$ Sin embargo, para Descartes el "Cogito» no me dice solamente que soy (por lo que no sería tampoco necesario: a pesar de todo, para ser engañado tengo que ser), sino también qué cosa soy, o sea, una cosa que piensa («res cogitans»), y que por tanto no tiene materia, contraponiéndose, en cambio, a todo lo que la tiene, y por tanto es extenso («res extensa»). Por ello, Descartes, basándose en el "Cogito», identifica la materia con la extensión y, de este modo, reduce todas las propiedades materiales a las meras propiedades geométricas, y, por consiguiente, todas las mutaciones físicas al movimiento.

Hay que decir que en esta forma extrema el mecanicismo nunca fue adoptado por la ciencia, pues en efecto una materia sin ninguna propiedad cualitativa ni siquiera puede ser pensada (exactamente lo opuesto a lo que creía Descartes) y en todo caso no corresponde a la del mundo real.

14 La hipótesis del diablo engañador fue introducida explícitamente solo más tarde, en las Meditaciones metafísicas (Descartes 1641), pero la lógica fundamental de su razonamiento siempre siguió siendo la misma. 
De todos modos, el punto esencial para nuestro razonamiento es que todo esto pasa porque al principio Descartes decide basarse solo en una razón autosuficiente, toda cerrada en sí misma y que no reconoce ningún valor a la experiencia, como declara explicitamente al principio de su Discurso: «Luego, pues los sentidos a veces nos engañan, quise suponer que ninguna cosa fuese tal cual nos la hacen imaginar» (Descartes 1637a: 312). Entonces, el fundamento último de su mecanicismo está en su racionalismo, y no en cualquier motivo de tipo científico.

Esto no es nada casual, ya que entre las dos cosas hay un nexo profundo: una razón desligada de la experiencia tiende naturalmente a una concepción mecánica de la realidad. Si no fuese así, en efecto, la realidad no sería completamente dominable por la razón misma y por lo tanto debería admitir el imprevisto, que, por definición, solo puede ser conocido a través de la experiencia.

Sin embargo, cabe subrayar que todo esto no nace, como generalmente se dice, de la confianza en la razón, sino, en primer lugar, de una profunda desconfianza en la experiencia, que llega hasta la negación del nexo originario entre la razón y la experiencia. Esto es lo que se puede considerar el verdadero «dogma central» de la modernidad, es decir, el punto de origen común a todas las diferentes y a veces aparentemente opuestas filosofías que han nacido en los últimos 4 siglos: o sea, la invencible convicción que la razón nunca puede encontrar la verdad dentro de la experiencia.

En efecto, si adoptamos esta clave de lectura, muchas cosas que antes podían parecer paradójicas o incomprensibles se hacen claras. Por ejemplo, las dos posturas filosóficas más características de la modernidad, el racionalismo y el relativismo, aparentemente opuestos entre sí, ahora se revelan ser nada más que las dos caras de una misma medalla: caemos en el primero si no queremos renunciar a la verdad (pero luego hay que tirar la 
experiencia por la ventana); caemos en el segundo si no queremos renunciar a la experiencia (pero luego hay que tirar por la ventana la verdad). Más generalmente, toda la historia de la filosofía moderna se puede ver como una continua oscilación entre estos dos polos que Descartes separó primero y que ninguno depués de él quizo o pudo juntar de nuevo: por ello, cuando prevalece la tendencia hacia la valorización de la experiencia se abre una fase dominada por filosofías empiristas; cuando en cambio prevalece la tendencia hacia la valorización de la razón se abre una fase dominada por filosofías idealistas; en el medio, a veces se pasa por una fase caracterizada por un dualismo inestable, como el de Descartes mismo o el de Kant, durante la cual los dos aspectos coexisten sin ser realmente unidos; ${ }^{1} \mathrm{y}$ después, todo empieza de nuevo igual. Y hasta cuando no se encontrará el coraje de rechazar el dogma central, siempre todo irá a empezar igual de nuevo, y de nuevo, y de nuevo, según un proceso bien conocido por los psicólogos con el nombre de «coacción a repetir». Y en efecto hay algo patológico en todo esto, ya que nace de una postura no serena frente a la realidad, como veremos de pronto.

1 Y otras veces no. Llamo «transición de tipo Kant» el primer caso, pues Kant fue el primero y el más celebre en proponer esta solución después del "cisma» cartesiano; y «transición de tipo Berkeley» el segundo, en que empirismo e idealismo se convierten directamente uno en otro, pues George Berkeley (1685-1753), por su famoso principio "Esse est percipi» (Cf. Berkeley 1710), es considerado tanto un empirista como un idealista por diferentes autores $-\mathrm{y}$ a veces incluso por el mismo autor-. Claro que todo esto no se debe considerar como una ley intrínsica a la historia del pensamiento como tal, lo que sería de nuevo una foma de idealismo y, por tanto, lo más ajeno de mis intenciones que se pueda imaginar. Yo no digo que hay una necesidad fatal por la cual las cosas tengan que desarrollarse de una cierta manera: pero sí digo que si se aceptan ciertos supuestos, luego derivan necesariamente ciertas consecuencias. Ni tampoco digo que en cada momento histórico todos los filósofos estén costringidos a tomar una cierta postura (lo que, dejando de lado toda teoría, sería simplemente falso de hecho): pero sí digo que en cada momento histórico hay una cierta postura filosófica que prevalece, y no acaso, sino siempre por razones precisas (aunque no siempre buenas). 


\subsection{Duda Metodológica y EXPERIENCIA DEL RIESGo}

Es muy instructivo ver como Descartes finaliza su posición, y por qué. En efecto, él continúa afirmando que también todas las otras ideas que tenemos y que son tanto «claras y distintas» como el «Cogito» tienen que ser verdaderas, porque si fueran falsas nunca podríamos darnos cuenta, y Dios, siendo bueno, no puede permitir que seamos engañados sin remedio. Sin embargo, las tres demostraciones de la existencia de Dios que Descartes propone están todas basadas en el supuesto que las ideas «claras y distintas» son verdaderas, por tanto caen en un círculo vicioso (Cf. Descartes 1641: 33-49). Lo interesante aquí es que el problema básico que afecta su razonamiento es el mismo que empujó a Galileo a actuar su revolución metodológica y que, como hemos visto, Descartes en cambio rechazó, o sea, que el mundo es contingente: por consiguiente, o lo aceptamos como un dato originario e irreductible (podríamos decir también como un don), o lo perdemos sin remedio, ya que eso no puede ser «deducido» por ningún principio lógico o metafísico, tanto por lo que concierne sus propiedades particulares como por lo que le corresponde a su misma existencia.

Es claro que entre las dos cosas hay una estrecha relación, pero que, antes de ser teorética, es, en primer lugar, psicológica y existencial. Aceptar al mundo como un dato, en efecto, obliga a fiarse de algo que no se puede controlar, pues es otro de uno mismo, y por tanto a hacer la experiencia del riesgo. Por tanto, en la base de todo está una opción fundamental que involucra toda la persona y su postura, abierta o cerrada, frente a toda la realidad. Claramente, dicha opción no involcura solo la razón, sino también la libertad: sin embargo, una postura es razonable y otra no. Esto no se puede demostrar formalmente (pues en este caso no 
se trataría de una opción fundamental, ya que toda demostración tiene que basarse en algo más fundamental, y por tanto las cosas más fundamentales de todas no se pueden demostrar por supuesto), pero se puede mostrar mirando sus consecuencias. Y, en efecto, quien acepta este incial «salto en el vacío», de pronto descubre la posibilidad de una gran fecundidad, mientras quien no quiere moverse sin «seguro», para así decirlo, se encontra condenado a la esterilidad. Esto recuerda el dicho del Evangelio «Quien quiere salvar a su vida la perderá, y quien la pierda por mí la encontrará» ( $L c$ 9, 24), y no vale solo en la vida personal, sino también en la ciencia. En efecto, también uno de los científicos más distinguidos del siglo XX como Max Planck (1858-1947) en su obra El conocimiento del mundo fisico menciona algo similar, aunque ciertamente no pensaba en Descartes cuando lo escribió: «No nos vengan a decir que hasta en la más exacta de todas las ciencias se pueda proceder sin una intuición del mundo, o sea, sin hipótesis indemostrables. También en la física no se puede ser beatos sin la fe, por lo menos sin la fe en una realidad fuera de nosotros» (Planck 1933: 77-78). E incluso un científico que no creía en Dios, o por lo menos en un Dios personal, como Werner Heisenberg (1901-1976), en el momento de su descubrimiento más grande no pudo evitar de hablar de la realidad como un don: «Y ahora [...], todo el territorio de las relaciones internas de la teoría atómica se ha desplegado repentinamente ante mis ojos con claridad. Que estas relaciones internas muestren, en toda su abstracción matemática, un grado de increíble sencillez, es un don que solo podemos aceptar con humildad. Ni siquiera Platón habría podido creer que fueran tan bellas. Estas relaciones, en efecto, no pueden ser inventadas. Existen desde la creación del mundo» (Heisenberg 1971: 71-72).

Luego, tampoco la "duda metodológica» de Descartes no tiene nada que ver con el auténtico método científico: más bien, nace precisamente de su malentendimiento, y llega inevitablemente a un escepticismo absoluto, y 
no solo metodológico (Cf. Musso 2011). Sin embargo, cabe subrayar que esta no era la intención de Descartes, para el cual la duda servía solo para llegar a una certeza más fuerte y, de todos modos, no era por nada el método de todo conocimiento, sino solo de su fase inicial y «fundativa». Luego, es extremadamente improbable que Descartes, si viviera hoy, se reconocería en los actuales teóricos de la duda universal como única vía al pensamiento crítico y racional, aunque exactamente esto sea el lógico e inevitable desemboque de la tradición filosófica que ha fundado.

\subsection{MeCANiCismo Científico y MeCANiCISMo FILOSÓfico}

Como ya hemos dicho, por casi dos siglos el éxito espectacular de la ciencia, especialmente de la teoría de la gravitación universal de Newton (Cf. Newton 1687), pareció justificar el mecanicismo filosófico de tipo cartesiano. En efecto, el sistema newtoniano era ciertamente mecanicista por muchos aspectos, pues preveía la existencia de un Universo infinito, caracterizado por un espacio y un tiempo absolutos (es decir, que existían independientemente de los cuerpos), en que se movían los cuerpos que interactuaban entre sí, por contacto o por la gravedad (más tarde también por otras fuerzas que actuaaban de manera análoga, la eléctrica y la magnética). En cambio, por más extraño que pueda parecer, por sí misma la teoría newtoniana no preveía una teoria atómica de la materia, aunque sea fácil y hasta natural insertarla, como de hecho aconteció más tarde. Sin embargo, entre el mecanicismo filosófico y el científico hay por lo menos tres importantes diferencias:

1) En primer lugar, el mecanicismo científico de tipo newtoniano no era «completamente» mecánico: Newton rechazó la identificación cartesiana de la materia con la extensión y la pura forma geométrica, pues 
tenía también otras propiedades (masa y gravedad), y además la gravedad actuaba a distancia y no por contacto.

2) En segundo lugar, el mecanicismo científico era sencillamente un modelo particular, bueno para describir «algunas propiedades» de la naturaleza, según la lección galileana; mientras que el mecanicismo filosófico era una teoría metafísica, que pretendía valer de un modo absoluto.

3) Por último, el mecanicismo científico hacía afirmaciones controlables experimentalmente, que permitían corregirlo si necesario, como en efecto aconteció después.

Como instrumento para la formulación de teorías científicas, el mecanicismo es muy potente y útil. Solo que, como todas las cosas humanas, tiene sus límites. Por tanto, el error que tenemos que evitar es precisamente no reconocer estos límites, pretendiendo convertir a un modelo particular en una verdad metafísica, es decir, en una teoría filosófica y ya no científica, como lo quería Descartes y como lamentablemente aconteció a menudo en los siglos XVIII y XIX, y a veces sigue aconteciendo todavía hoy, también por obra de científicos distinguidos. Sin embargo, a pesar de la gran difusión de estas incorrectas interpretaciones, el mecanicismo científico, a diferencia del filosófico, conservó intacta su capacidad de autocorrección, gracias a su estructural apertura a la realidad, debida al método experimental de Galileo, que es como la «encarnación» operativa de su idea abierta de razón, y que en el tiempo se impuso contra los prejuicios de los científicos e incluso contra su propio (y extraordinario) éxito. En efecto, en el siglo xx las revoluciones de la relatividad, de la mecánica cuántica y, más tarde, del caos determinístico empujaron a la ciencia más allá del modelo newtoniano, es decir, hacia una concepción de la materia y de la energía que ya no puede, de ninguna manera, ser traducida en términos mecánicos (Cf. Musso 2011). 


\section{EL ALBA INCONCLUSA DEL RENACIMIENTO}

Según la interpretación dominante, en el Renacimiento nació, de Galileo y Descartes, una nueva idea de razón, el racionalismo, y una nueva cultura, la modernidad.

Sin embargo, como hemos visto, lo que realmente ocurrió fue una dramática dicotomía entre dos opuestas concepciones de la razón y de la cultura, una sostenida por Descartes (la del racionalismo) y una por Galileo (que seguía y potenciaba, abriéndole nuevas perspectivas, la tradición clásica y cristiana), cada una de las cuales causará en los siglos siguientes profundísimas consecuencias en muchos otros aspectos de la concepción del hombre y de su actuar en el mundo (Cf. Musso 2011: 208-214 y 533-548).

Quizás la más dramática consecuencia de la posición de Descartes es el dualismo metafisico, es decir, la introducción de una drástica separación entre el mundo de la materia y el del espíritu. En efecto, Dios parece importante - $\mathrm{O}$ más bien fundamental - en el sistema de Descartes, pero en realidad este Dios sirve solo para asegurarnos que el mundo existe (a pesar de que, como hemos visto, al final ni siquiera lo logra), pero, por lo demás, el mundo es un sistema mecánico separado y autosuficiente, en que Dios no hace nada. Como dijo el filosofo italiano Cornelio Fabro, con Descartes empieza aquella tendencia de la mentalidad moderna por la cual «Dios, si existe, no importa» (Cf. Fabro 1969). Y podríamos añadir: «y el alma tampoco», ya que lo mismo vale para el alma, que por una parte tiene en sí misma toda verdad, pero, por otra, es completamente separada del cuerpo, que es también autosuficiente y que se comunica solo a través de la misteriosa glándula pineal, que es como la «encarnación» de la fragilidad de esta filosofía. 


\section{ENSANCHAR LA RAZÓN}

Todo esto no tiene solo consecuencias filosóficas y teóreticas, sino también existenciales, ya que en dicha perspectiva no solo mundo y Dios, cuerpo $y$ alma, sino también sentimiento y razón, conocimiento y realidad, subjetividad y objetividad coexisten, pero sin estar realmente juntos, sino separados en raíz. Esta herencia mucho se relaciona con el dramático sentimiento de división interior y de fragilidad personal que el hombre moderno muy a menudo experimenta. Pero - $\mathrm{y}$ este es el punto- esta herencia no es un destino fatal, que deriva necesariamente del progreso del conocimiento y de la ciencia como tales, como casi siempre se dice. Al contrario, esta es una completa contrafacción de la verdadera modernidad, que nació de la ciencia experimental y por tanto es la de Galileo (y no la de Descartes), y por ello implica una razón originariamente connexa a la experiencia (y no desligada de ella), un pluralismo metodológico (y no el reduccionismo) y, sobre todo, una infinita apertura de la razón al imprevisto y al misterio (y no una razón mecánica y cerrada en sí misma).

Luchar en contra de esta contrafacción no será una tarea ni simple ni corta, considerando la difusión y el soporte que tiene hoy en día en el mundo. Pero tenemos un aliado muy valiente, Galileo, y un instrumento muy poderoso, la ciencia, la cual, a pesar de los prejuicios contrarios que desgraciadamente hoy muchos científicos tienen (pues, a pesar de todo, también los científicos son hombres de su tiempo), nunca podrá llevarse bien con una concepción reduccionista y mecánica de la razón, y por tanto siempre seguirá pidiendo su continuo ensanche. 


\section{BIBLIOGRAFÍA}

BANFI, Vittorio

2007 «Isaac Newton vs René Descartes». Emmeciquadro, año 2007, N. 30, pp. 35-42.

Bellarmino, Roberto

[1615] Lettera a Paolo Antonio Foscarini, 12 aprile 1615, manuscrito; en Edizione Nazionale delle Opere di Galileo Galilei. Firenze: Giunti Barbera, 1890-1909, XII, pp. 171-172.

Berkeley, George

1710 A treatise concerning the principles of human knowledge. Dublin: Aaron Rhames.

Descartes, René

1628 Regulae ad directionem ingenii, manuscrito; publicación póstuma 1701, Blaeu, Amsterdam; trad. it. Regole per la guida dell'intelligenza. En Opere filosofiche. Tomo I. Bari: Laterza, 1986, pp. 15-94.

1633a Le Monde ou Traité de la Lumière, publicación póstuma 1677, Clerselier, Paris; trad. it. Il Mondo o Trattato della luce, en Opere filosofiche. Tomo I. Bari: Laterza, 1986, pp. 123-201.

1633b L'Homme, manuscrito; publicación póstuma 1677, Clerselier, Paris; trad. it. L'Uomo, en Opere filosofiche. Tomo I. Bari: Laterza, 1986, pp. 203-287. 
PAOLO MUSSO

1637a Discours de la Méthode, Leida; trad. it. Discorso sul metodo, en Opere filosofiche. Tomo I. Bari: Laterza, 1986, pp. 289-342.

1637b La dioptrique, Leida; trad. it. La diottrica, en Opere scientifiche. Tomo II. Bari: Laterza, 1983, pp. 185-345.

1638 Lettre à Marin Mersenne, 11 Octobre 1638, manuscrito. En Edizione Nazionale delle Opere di Galileo Galilei. Firenze: Giunti Barbera, , 1890-1909, XVII, pp. 387-391.

1644 Principia philosophiae, Elsevier, Amsterdam; trad. it. I principi della filosofia. En Opere filosofiche. Bari: Laterza, 1986, III, pp. 1-393.

Einstein, Albert

1934 Mein Weltbild, Querido, Amsterdam; trad. ing. The World as I See It. New York: Simon \& Schuster; trad. it. Come io vedo il mondo. Milano: Cucchini, 1955.

Einstein, Albert e Leopold Infeld

1938 The Evolution of Physics. The Growth of Ideas from Early Concepts to Relativity and Quanta, New York: Simon \& Schuster; trad. it. L'evoluzione della fisica. Sviluppo delle idee dai concetti iniziali alla relatività e ai quanti. Torino: Boringhieri, 1948.

FABro, Cornelio

1969 Introduzione all'ateismo moderno. Roma: Studium. 
Galilei, Galileo

1613 Istoria e dimostrazioni intorno alle macchie solari e loro accidenti, Giacomo Mascardi, Roma. En Edizione Nazionale delle Opere di Galileo Galilei. Firenze: Giunti Barbera, 1890-1909, V, pp. 72-249.

1615 Lettera a Madama Cristina di Lorena, manoscritto. En Edizione Nazionale delle Opere di Galileo Galilei, Giunti Barbera, Firenze, 1890-1909, V, pp. 309-348.

1632 Dialogo sopra idue massimi sistemi del mondo, tolemaico e copernicano, Giovanni Battista Landini, Firenze. En Edizione Nazionale delle Opere di Galileo Galilei, Giunti Barbera, Firenze, 1890-1909 VII, pp. 23-546.

Heisenberg, Werner

1971 Physics and beyond: encounters and conversations, Harper \& Row, New York; trad. it. Fisica e oltre. Incontri con i protagonisti, 19201965. Torino: Bollati Boringhieri, 1984.

KePler, Johannes

1611 Dioptrice. Augusta: David Frank.

Laplace, Pierre-Simon de

1776 Essai philosophique sur les probabilités. Paris: Bachelier; trad. it. Saggio filosofico sulle probabilità. En Opere. Torino: UTET, 1967, pp. 241-404. 
Musso, Paolo

2011 La scienza e l'idea di ragione. Scienza, filosofia e religione da Galileo ai buchi neri e oltre. Milano-Udine: Mimesis.

Newton, Isaac

1687 Philosophiae naturalis principia mathematica, Joseph Streater, London; trad. it. Principi matematici della filosofia naturale. Torino: UTET, 1965.

Planck, Max

1933 Wege zur physikalischen Erkenntnis. Reden und Vörtrage, S. Hirzel, Leipzig; trad. it. La conoscenza del mondo fisico. Torino: Boringhieri, 1954. 